\title{
Finite Element Analysis of Cylindrical Rubber Fender
}

\author{
Chung-Ming Tan ${ }^{1,}$, Mau-Yiu Chang ${ }^{1}$ \\ ${ }^{1}$ Graduate School of Opto-Mechatronics and Materials, WuFeng University, ChiaYi, Republic of China
}

\begin{abstract}
This research is to develop a methodology to optimize the performance of fenders using finite element analysis. The design of fender geometry is done using software SolidWorks. Simulation is one of several add-in tools in SolidWorks. The finite element analysis of the performance of the fender designed is then evaluated by simulation tool in SolidWorks. This study also shows the potential applications of computer aided engineering and its benefits in verifying and reinventing various fenders design.
\end{abstract}

\section{Introduction}

The berthing of ship to the jetty is the last stage of navigation process in marine shipping. During the ships berthing the impact in berth can occur and in result the appearing of energy in ship - fender device - berth system [1-6]. The exceeding of admissible value of this energy leads to damage of one of these elements and in effect causes the accident. The accident can happen as unwanted event with negative consequences such as a damage of ship and port structure. The fenders are devices to protect the port structures and vessel from damage by absorbing the kinetic energy of vessel while vessel berthing to the jetty or quay [7-9]. For this study, the performances of fender system are analyzed using finite element analysis model. The finite element method is used to analyze the performance of a cylindrical rubber fender system when subjected to the impact load caused by vessel collision. The cylindrical fender system is one of the buckling type fenders which exhibit good energy absorption capacity during post-buckling deformation. The inner diameter of a hollow cylindrical fender is chosen to be the parameter which will be optimized with the aid of finite element analysis.

\section{Material and Methodology}

Two phenomenological models for hyperelastic materials - the Neo-Hookean, Mooney-Rivlin-are predefined in the SolidWorks Material models. One can use both the Neo-Hookean and the Mooney-Rivlin material models for modeling nearly incompressible hyperelastic materials such as rubber. Extracting the parameters for the material models from experimental stress strain curves involves curve fitting with the appropriate equations for the nominal (Piola-Kirchhoff) stress. For a thin sheet of incompressible hyperelastic material under uniaxial tension the stretch along the axis

\footnotetext{
a Corresponding author: cmtan@wfu.edu.tw
}

of the loading is $\lambda_{1}=\lambda=1+\varepsilon$, where $\varepsilon$ is the strain. Due to symmetry and incompressibility the stretch ratios in the transverse directions are $\lambda_{2}=\lambda_{3}=\lambda-1 / 2$. The stress state corresponding to this state of deformation is reduced to $\mathrm{P}_{1}=\mathrm{P}, \mathrm{P}_{2}=\mathrm{P}_{3}=0$, where

$$
\mathrm{P}=2\left(\lambda-\lambda^{-2}\right)\left(\frac{\partial W_{h y p}}{\partial I_{1}}+\frac{1}{\lambda} \frac{\partial W_{h y p}}{\partial I_{2}}\right)
$$

Assume a strain energy function, $\mathrm{W}_{\mathrm{hyp}}$, according to the Mooney-Rivlin material model, for which Equation (1) becomes

$$
\mathrm{P}=2\left(\lambda-\lambda^{-2}\right) C_{10}+2\left(1-\lambda^{-3}\right) C_{01}
$$

Then use Equation (2) to curve fit the parameters $\mathrm{C}_{10}$ and $\mathrm{C}_{01}$ from a uniaxial stress-strain curve.

\subsection{Computer Aided Engineering Methodology}

In this study, we have designed a three-phase structure which consists of initial CAD model, FEA performance evaluation and CAD model modification as shown in figure 1. In this study, we optimize the inner diameter of a hollow cylindrical rubber fender.

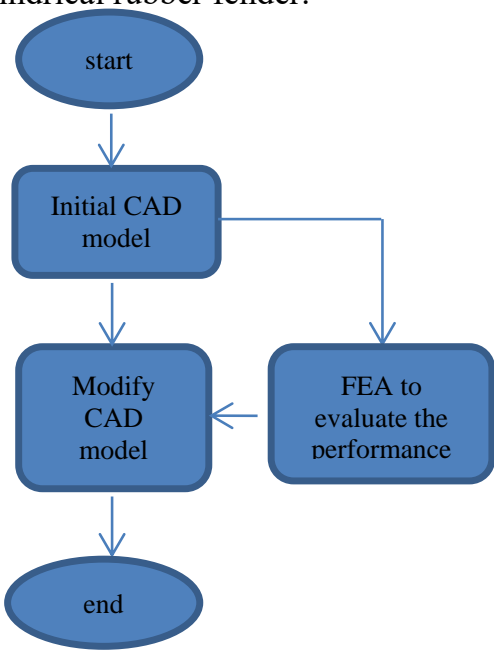


Figure 1. The flowchart of the parameter study process in this study.

\subsection{Finite Element Analysis}

The software uses the Finite Element Method (FEM). FEM is a numerical technique for analyzing engineering designs. FEM is accepted as the standard analysis method due to its generality and suitability for computer implementation. FEM divides the model into many small pieces of simple shapes called elements effectively replacing a complex problem by many simple problems that need to be solved simultaneously.

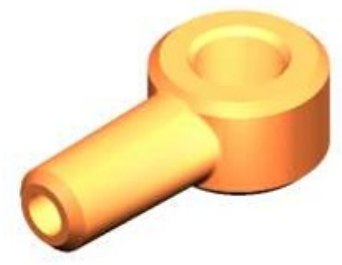

CAD model of a part

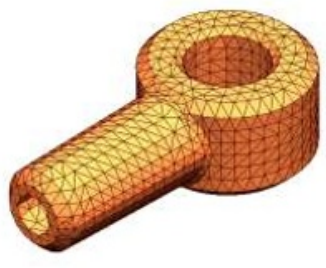

Model subdivided into small pieces (elements)
Figure 2. Illustration of meshing in finite element analysis.

Elements share common points called nodes. The process of dividing the model into small pieces is called meshing as shown in the figure 2 . The behavior of each element is well-known under all possible support and load scenarios. The finite element method uses elements with different shapes. The response at any point in an element is interpolated from the response at the element nodes. Each node is fully described by a number of parameters depending on the analysis type and the element used. For example, the temperature of a node fully describes its response in thermal analysis. For structural analyses, the response of a node is described, in general, by three translations and three rotations. These are called degrees of freedom (DOFs). Analysis using FEM is called Finite Element Analysis (FEA).

In nonlinear finite element analysis, a major source of nonlinearities is due to the effect of large displacements on the overall geometric configuration of structures. Structures undergoing large displacements can have significant changes in their geometry due to loadinduced deformations which can cause the structure to respond nonlinearily in a stiffening and/or a softening manner. There are different numerical procedures that can be incorporated in the solution of nonlinear problems using the finite element method. A successful procedure must include the following:

-A control technique capable of controlling the progress of the computations along the equilibrium path (s) of the system.

- An iterative method to solve a set of simultaneous nonlinear equations governing the equilibrium state along the path (s).

-Termination schemes to end the solution process.
Additional schemes such as line search, acceleration, and/or preconditioning may be augmented to enhance the solution procedure. In this study, we adopted the add-in tool called "simulation" in SolidWorks to implement the nonlinear finite element analysis described roughly in this section.

\section{Results and Discussion}

The initial CAD design for the cylindrical fender is represented by a hollow cylinder which has the height of $100 \mathrm{~mm}$, outer diameter of $100 \mathrm{~mm}$, and inner diameter of $50 \mathrm{~mm}$. Then we started using SolidWorks to create a hollow cylinder and employed the simulation tool to undergo the finite element analysis. One end of the cylinder is set to be fixed and the other end is set to have a prescribed displacement of $60 \mathrm{~mm}$ to compress the cylinder. From the experimental data of an actual tension test, the Mooney-Rivlin coefficient $\mathrm{c} 01$ and $\mathrm{c} 10$ can be found using the above mentioned equation (2). By fitting these data to the equation, the coefficients $\mathrm{C} 01$ and $\mathrm{C} 10$ of Mooney-Rivlin model used in the simulation are 1.7 Mpa and 0.55 Mpa respectively. The stress flooded plot at maximum compression displacement and the reaction forces versus compression displacement curves for the initial CAD model are presented in the figure 3.

From figure 3 , it is obvious that buckling of the whole fender occurred around the pseudo time of 0.68 second. The pseudo time 1 second here stands for how long it would take when the whole prescribed displacement at one end is completed in the simulation. The simulation is terminated at pseudo time of 0.86 second. This is due to the structural instability induced by the local buckling at both ends. Another reason for termination is that selfcontact condition at both ends also failed.

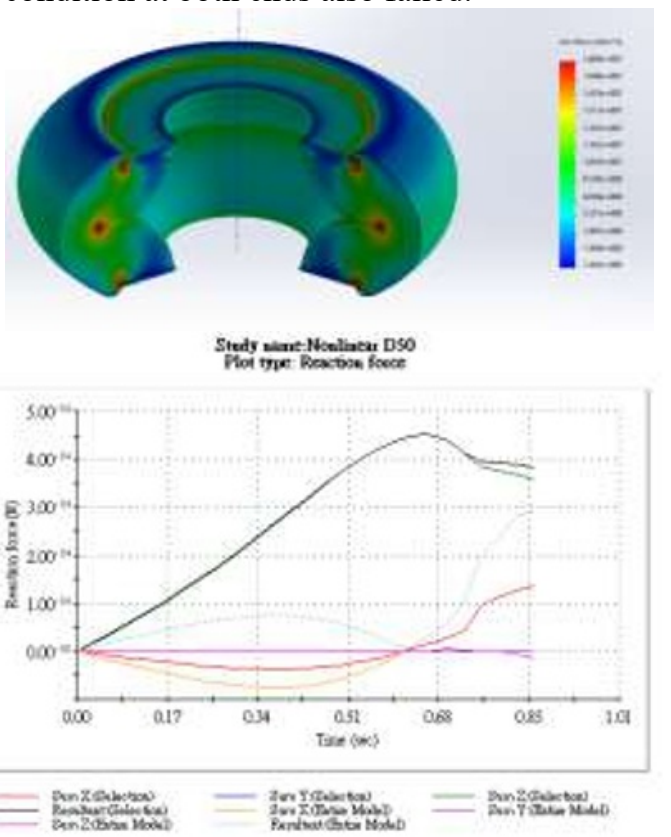

Figure 3. The stress flooded plot at maximum compression displacement and the reaction forces versus compression displacement curves for inner diameter of $50 \mathrm{~mm}$.

In order to improve the result of the initial CAD model, we varied the inner diameter by increment of 5 
$\mathrm{mm}$ at one time and repeated the simulation by 4 times till the inner diameter reaches $70 \mathrm{~mm}$. Simulation results for different inner diameters are presented in 4 following figures. First, we compared the results in figures 3 and 4. Global buckling behavior and local buckling at both ends are similar. Therefore, no obvious better performance can be seen except the axial stiffness decreased by $20 \%$ or so.
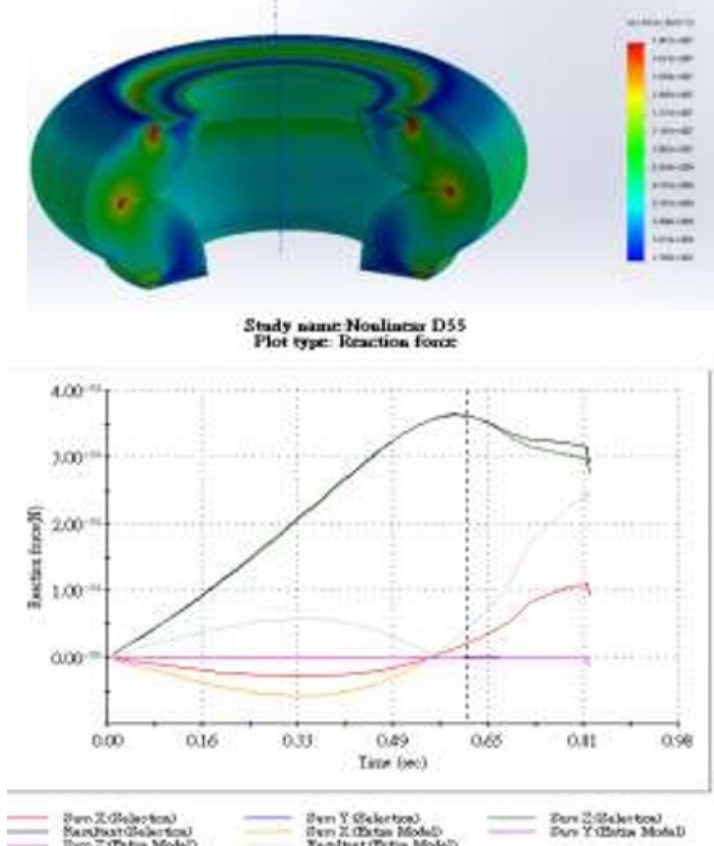

Figure 4. The stress flooded plot at maximum compression displacement and the reaction forces versus compression displacement curves for inner diameter of $55 \mathrm{~mm}$.

From figure 5, one can easily tell that the postbuckling behavior observed can be improved the energy absorption capacity a lot. The reaction force versus displacement curve revealed favorable s-shape. This means that post-buckling has a large region maintains high reaction force that improves the energy absorption capacity. Fenders consisting of buckling elements are connected in a manner assuring its desired characteristic. This generally features rapid growth of reaction force at small deflection, and then the reaction remains more or less the same although deflection increases. Examining strength characteristics of fender materials, engineers attempt to obtain possibly small elasticity constant with possibly large relative elongation. Such material should have high level of maximum admissible stresses. The vessel loses its speed and kinetic energy by deforming a fender along a certain distance. The vessel and accompanying water have a certain mass when berthing at a certain speed and hitting the fender. Therefore, it can be said that the measure of fender elasticity is the length of elastic deflection caused by a certain force. In practice, relative deflection (percentage) is used.

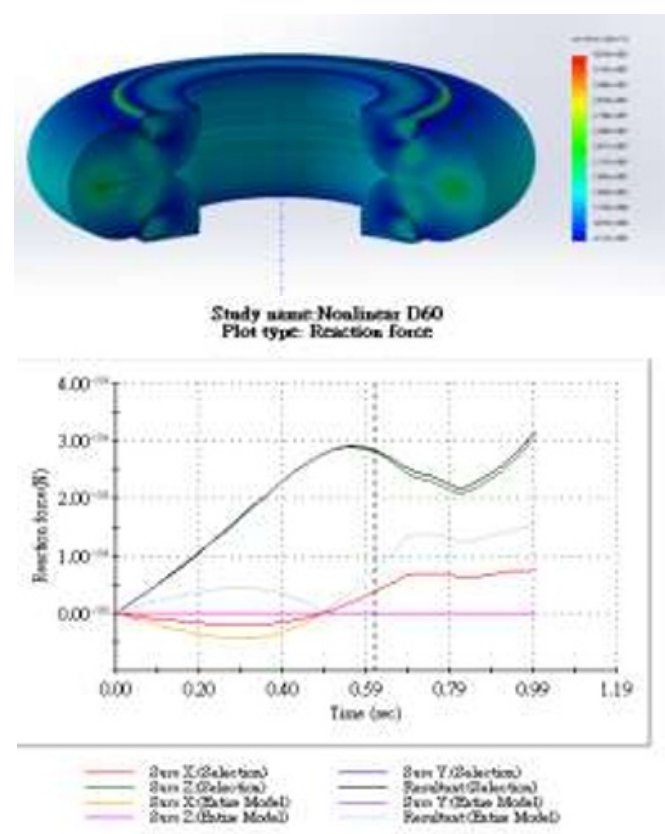

Figure 5. The stress flooded plot at maximum compression displacement and the reaction forces versus compression displacement curves for inner diameter of $60 \mathrm{~mm}$.

Further increase of the inner diameter, figure 6 revealed the further improvement but figure 7 revealed no improvement can be seen. Comparing the figures 6 and 7 , we noticed that there is a further force drop happened during the post-buckling for inner diameter of $70 \mathrm{~mm}$. The phenomenon stops the improvement of the energy absorption.

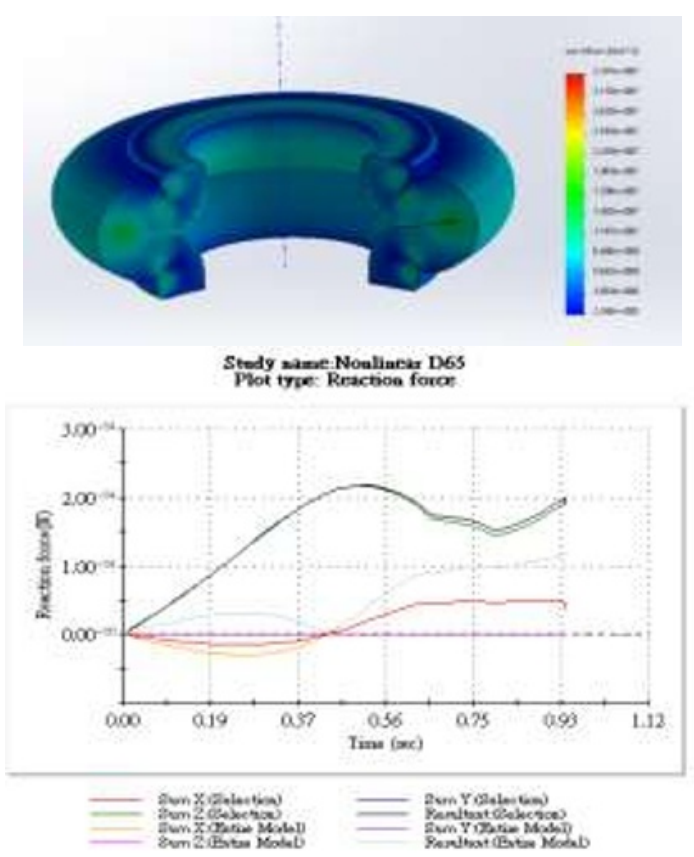

Figure 6. The stress flooded plot at maximum compression displacement and the reaction forces versus compression displacement curves for inner diameter of $65 \mathrm{~mm}$. 


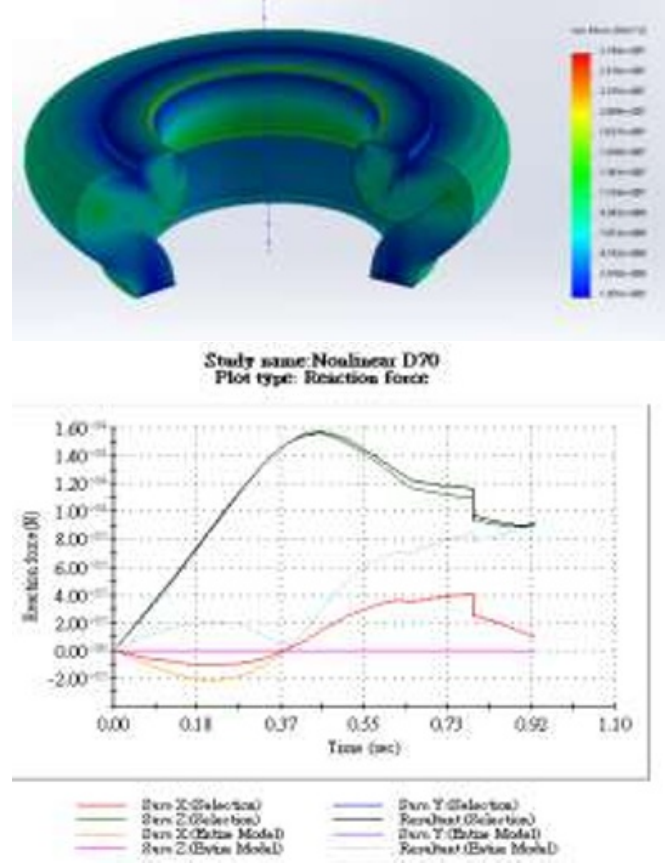

Figure 7. The stress flooded plot at maximum compression displacement and the reaction forces versus compression displacement curves for inner diameter of $70 \mathrm{~mm}$.

\section{Conclusion}

This research showed the potential applications of computer aided engineering and its benefits by optimizing the performance of a hollow cylindrical rubber fender. The initial CAD design was drawn in SolidWorks. Using the add-in simulation tool in SolidWorks, one can implement the nonlinear finite element analysis to evaluate the postbuckling behavior of a hollow cylindrical rubber fender. Choosing the inner diameter to be the parameter, we can apply the iterative methodology to maximize the energy absorption capacity of the fender. Finally an optimal inner diameter was accomplished which is between $65 \mathrm{~mm}$ and $70 \mathrm{~mm}$. The research specially emphasizes the implementation of computerized simulation which ultimately saved a lot of time and loss of material and gave an excellent result.

\section{References}

1. M. N. Hamza, H. M. Alwan, Eng. \&Tech. J. 23 25602575 (2010)

2. A. Chegenizadehi, B. Ghadimil, H. Nikrazi, E. Heshmati, J. Eng. Sci. Tech. 101054 - 1064 (2015).

3. W. Galor, J. JONES powertrain transp. 14 169-175 (2007).

4. G. Neser, D. Unsalan, Ocean Eng. 33 1919-1934 (2006).

5. M. Zikra1, K. Sambodho1, T. A. Manan, and Y. Mulyadi1, J. Eng. Appl. Sci. 11 896-900 (2016).

6. M. S. Zala, K. J. Motwani, N. J. Patel, Int. J. Adv. Eng. Res. Dev. 2 1144-1149 (2015).

7. S. Sakakibaraa, M. Kubob, Ocean Eng. 34 1174-1181 (2006).

8. Z. Jiang, M. Gu, Mat. Des. 31 1085-1095 (2009).
9. M. Gohil, U.V. Shah, Int. J. Sci. Res. $52277-8179$ (2013). 